\title{
Development of Fluorocarbon Evaporative Cooling Recirculators and Controls for the ATLAS Inner Silicon Tracker
}

\author{
C. Bayer ${ }^{1}$, S. Berry 2 , P. Bonneau ${ }^{2}$, M. Bosteels ${ }^{2}$, H. Burckhart ${ }^{2}$, D. Cragg 3 , R. English ${ }^{3}$, \\ G. Hallewell ${ }^{3,4+}$, B. Hallgren ${ }^{2}$, S. Ilie ${ }^{2}$, S. Kersten ${ }^{1}$, P. Kind ${ }^{1}$, K. Langedrag 5 , \\ S. Lindsay ${ }^{6}$, M. Merkel ${ }^{2}$, S. Stapnes ${ }^{5}$, J. Thadome ${ }^{1}$, V.Vacek ${ }^{2,7}$ \\ ${ }^{1}$ Physics Department, Wuppertal University, Germany; ${ }^{2}$ CERN, 1211 Geneva 23, Switzerland; \\ ${ }^{3}$ Rutherford Appleton Laboratory, Chilton, Didcot, OX11OQX, UK; \\ ${ }^{4}$ Centre de Physique des Particules de Marseille, Campus des Sciences de Luminy, 13288 Marseille, France; \\ ${ }^{5}$ Physics Department, Oslo University, Norway; 6 Physics Department, Melbourne University, Australia; \\ ${ }^{7}$ Czech Technical University, Prague, Czech Republic.
}

\begin{abstract}
We report on the development of evaporative fluorocarbon cooling recirculators and their control systems for the ATLAS inner silicon tracker.

We have developed a prototype circulator using a dry, hermetic compressor with $\mathrm{C}_{3} \mathrm{~F}_{8}$ refrigerant, and have prototyped the remote-control analog pneumatic links for the regulation of coolant mass flows and operating temperatures that will be necessary in the magnetic field and radiation environment around ATLAS.
\end{abstract}

Temperature, pressure and flow measurement and control use 150+ channels of standard ATLAS LMB ("Local Monitor Board") DAQ and DACs on a multi-drop CAN network administered through a BridgeVIEW user interface. A hard-wired thermal interlock system has been developed to cut power to individual silicon modules should their temperatures exceed safe values.

Highly satisfactory performance of the circulator under steady state, partial-load and transient conditions was seen, with proportional fluid flow tuned to varying circuit power. Future developments, including a $6 \mathrm{~kW}$ demonstrator with $\sim 25$ cooling circuits, are outlined.

\section{INTRODUCTION}

For a ten-year operational lifetime in the high radiation field close to the LHC beams, the silicon substrates of the ATLAS SCT and pixel detectors must operate below $\sim-6^{\circ} \mathrm{C}$, with only short warm-up periods each year for maintenance. Around $60 \mathrm{~kW}$ of heat will be removed through $\sim 400$ parallel cooling circuits. Evaporative fluorocarbon cooling has been chosen since it offers minimal extra material in the tracker sensitive volume, with refrigerants [in this work, per-fluoro-n-propane; $\left.\left(\mathrm{C}_{3} \mathrm{~F}_{8}\right)^{1}\right]$ that are non-flammable, non-conductive and radiation resistant (section $\mathrm{V}$ ).

\footnotetext{
${ }^{1}$ PF5030 Mfr.: 3-M Corp. Specialty Chemicals Division, St. Paul, MN55113-3223, USA
}

Following our earlier studies of evaporative cooling of ATLAS pixel and SCT thermo-structures [1,2], we have addressed the development of evaporative fluorocarbon recirculators, their control systems and services plant for use with $\mathrm{C}_{3} \mathrm{~F}_{8}$ at an evaporation temperature (pressure) of $\sim-25^{\circ} \mathrm{C}\left(\sim 1.7 \mathrm{bar}_{\text {abs }}\right)$. A prototype circulator (Figure 1) is centred on a hermetic, oil-less piston compressor ${ }^{2}$ operating at an aspiration pressure of $\sim 1$ bar $_{\text {abs }}$ and an output pressure of $\sim 10 \mathrm{bar}_{\text {abs. }}$.

\section{Prototype Circulator and Control System}

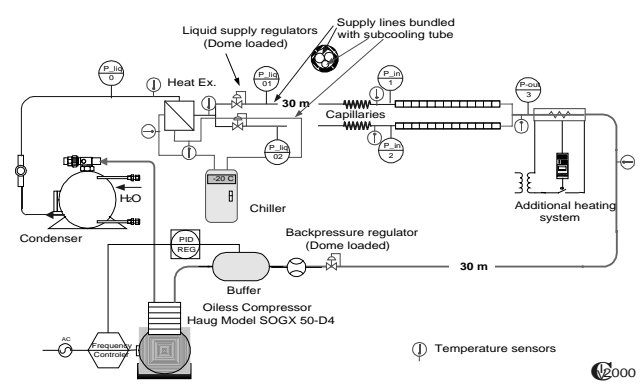

Figure 1: Schematic of Prototype Evaporative Recirculator

Aspiration pressure is regulated via PID variation of the compressor motor speed from zero to $100 \%^{3}$, based on the sensed pressure in an input buffer tank. A thermal simulator of 22 individually powered barrel SCT silicon strip modules is used as a variable load in these studies. Each module is monitored with a PT100. The thermal interlock to the module power supply is triggered from a negative temperature coefficient (NTC) ${ }^{4}$ thermal sensor.

\footnotetext{
2 Model SOGX 50-D4; Mfr: Haug Kompressoren CH-9015 St Gallen, Switzerland

${ }^{3}$ Via Motor Speed Controller: Model CIMR-XCAC41P5, (400V 3 phase, $3.7 \mathrm{kVA}$ ).

Mfr: Yaskawa Co., 1-16-1 Kaigan, Minato-Ku, Tokyo, 105-6891 Japan

${ }^{4}$ Semitec AT series: $10 \mathrm{k} \Omega @ 25^{\circ} \mathrm{C}, \mathrm{t}(\mathrm{K})=1 /(9.577 \mathrm{E}-4$

$\left.+2.404 \mathrm{E}-4 \ln (\mathrm{R})+2.341 \mathrm{E}-7 \ln (\mathrm{R})^{3}\right)$. Mfr: Ishizuka Electronics Co.

7-7 Kinshi 1-Chome, Sumida-ku, Tokyo 130-8512, Japan
} 


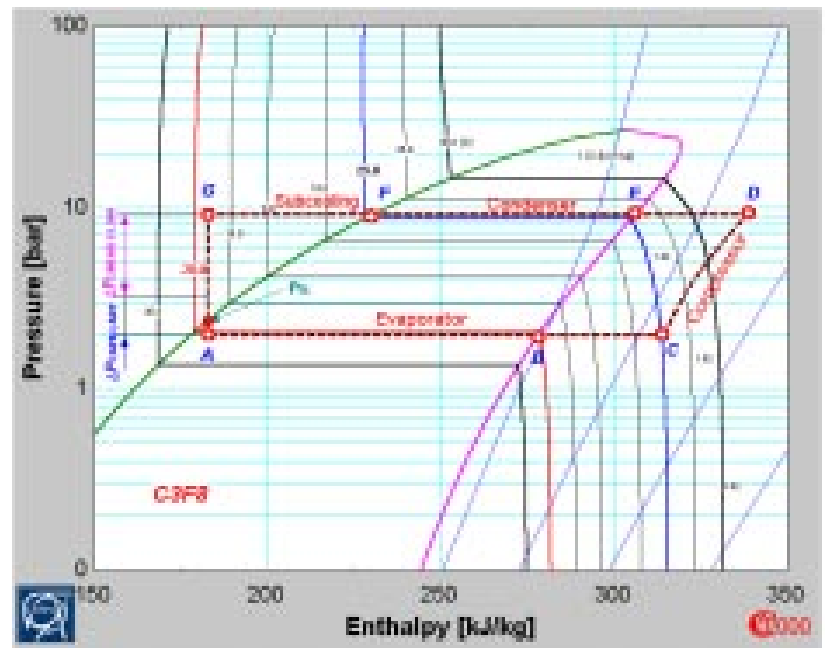

Figure 2: Cooling cycle on the P-h diagram

Figure 2 shows the closed thermodynamic cycle. High pressure $\mathrm{C}_{3} \mathrm{~F}_{8}$ vapor is condensed (EF) and passed to the detector loads in liquid form, at a flow rate dependent on the pressure upstream of injection capillaries with typical diameters $0.6-0.8 \mathrm{~mm}$. The refrigerative power depends on the product of mass flow rate $\left(\mathrm{kgs}^{-1}\right)$ and enthalpy $\left(\mathrm{Jkg}^{-1}\right)$. Since typical enthalpies are in the range $50-100 \mathrm{kJkg}^{-1}$ (depending on liquid sub-cooling), liquid flow rates of $\sim 10-20 \mathrm{gs}^{-1} \mathrm{~kW}^{-1}$ are typical. These flows are less than $\sim 1 / 20$ those typical in mono-phase liquid cooling systems, and allow reduced fluid service $\left(\% \mathrm{X}_{\mathrm{O}}\right)$ overhead close to the detector. The arrival temperature of liquid at the capillary also determines the available enthalpy. As an example in Figure 2, $100 \mathrm{kJkg}^{-1}$ (AB) is available with evaporation at $-20^{\circ} \mathrm{C}$ with $\mathrm{C}_{3} \mathrm{~F}_{8}$ subcooled to $-17^{\circ} \mathrm{C}$.

The higher the pressure available upstream of the capillary, the wider the proportional range of flow through it to accommodate circuit heat load variation (e.g. varying numbers of powered silicon detector modules); the zero flow limit being set by the saturated liquid pressure, $\mathbf{P}_{\mathbf{S L}}$. Since the maximum available pressure is delimited by the condenser pressure, reduction of the liquid arrival temperature (i.e. increased sub-cooling) tracks the saturated liquid locus to lower pressure $(\mathbf{F} \rightarrow \mathbf{A})$ and increases the linear flow range ( $\Delta \mathbf{P}_{\text {LINEAR FLOW }}$ ). Maximum enthalpy is possible when the liquid is subcooled to the evaporation temperature. For these reasons, the prototype recirculator of Figure 1 was equipped with variable temperature sub-cooling, (coolant tubes accompanied the $\mathrm{C}_{3} \mathrm{~F}_{8}$ liquid supply tube in the same insulated bundle), and with DAC-control of the liquid pressure upstream of the capillary.

The supply and return tubing of the cooling circuits must traverse the electrical services of other ATLAS subdetectors, which will be mainly located in an ambient air atmosphere with dew point $\sim 14^{\circ} \mathrm{C}$. These lines must therefore be insulated (and may require local surface heating in critical locations), to prevent condensation. Space for service passages is extremely limited, so efforts have been made to minimise insulation thickness by ensuring that as much as possible $(>90 \%)$ of the supplied $\mathrm{C}_{3} \mathrm{~F}_{8}$ liquid is evaporated in the on-detector tubing, through flow regulation proportional to circuit load. These studies are discussed in section III.B.

In the final installation, liquid will be distributed, and vapor collected from $\sim 400$ circuits by fluid manifolds on the ATLAS service platforms. This zone will inaccessible to personnel during LHC running, with local radiation levels and magnetic fields that exceed acceptable levels for a wide range of commercial control electronics. Local regulation devices will be mechanical; piloted with analog compressed air from electropneumatic ("E2P") actuators situated in an accessible zone.

Figure 3 illustrates the two-stage electropneumatic implementation tested in the prototype recirculator.

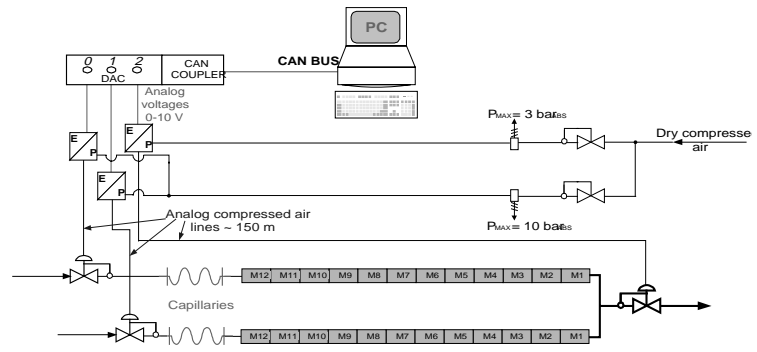

Figure 3: Electro-Pneumatic Control Schematic

Flow rate is proportional to the output pressure of a "dome-loaded" pressure regulator ${ }^{5}$ piloted by air in the range 1-10 bar $_{\text {abs }}$ from an E2P actuator ${ }^{6}$, which receives an analog set point from a $\mathrm{DAC}^{7}$. Circuit boiling pressure (hence operating temperature: e.g. at 1.9 bar $_{a b s}, \mathrm{C}_{3} \mathrm{~F}_{8}$ evaporates at $-20^{\circ} \mathrm{C}$ ) is controlled by a similarly piloted dome-loaded backpressure regulator ${ }^{8}$. In the final installation, the operating temperature of each parallel cooling circuit will be selectable through choice of evaporation pressure. Such individual control is impossible in a parallel-channel liquid cooling system with a single liquid supply temperature.

\footnotetext{
5 Model 44-2211-242-1099: Mfr: Tescom, Elk River MN 55330, USA

${ }^{6}$ Model PS111110-A: Mfr Hoerbiger Origa GmbH, A-2700 WienerNeustadt, Austria: Input 0-10V DC, Output pressure 1-11 bar ${ }_{\text {abs }}$

7 Model 750-556: Mfr Wago GmbH, D32423 Minden, Germany controlled through Model 750-307 CAN Coupler

${ }^{8}$ Model 26-2310-28-208: Tescom Corp
} 


\section{DATA ACQUISITION AND CONTROL SYSTEM ELECTRONICS}

\section{A. Data Acquisition}

Temperature, pressure and flow measurements in the circulation system, and on prototype SCT and pixel thermo-structures under test, are made using prototypes of the standard I/O system of the ATLAS DCS ("Detector Control System"). The industrial field-bus CAN [3] is used to read out distributed nodes, called LMBs ("Local Monitor Boards") [4], running the CANopen protocol [5]. The LMBs are designed to serve for the controls of all ATLAS sub-detectors, and will contain different analog and digital I/O functions. They are specified to tolerate a radiation dose of $1 \mathrm{~Gy}$ and $10^{11}$ neutrons $\mathrm{yr}^{-1} \mathrm{~cm}^{-2}$ and a magnetic field of $1.5 \mathrm{~T}$, and can hence be placed everywhere in the ATLAS experimental cavern outside the calorimeter. In the present system, $6 \mathrm{LMBs}$, each with up to 64 analog inputs multiplexed onto a 16 bit $\mathrm{ADC}$, are read out. The ADC range, polarity, and digitisation frequency are set through the CAN bus. The individual channels are mapped onto an ad-hoc implementation of a multiplexed PDO, inspired by the initial proposal in [6].

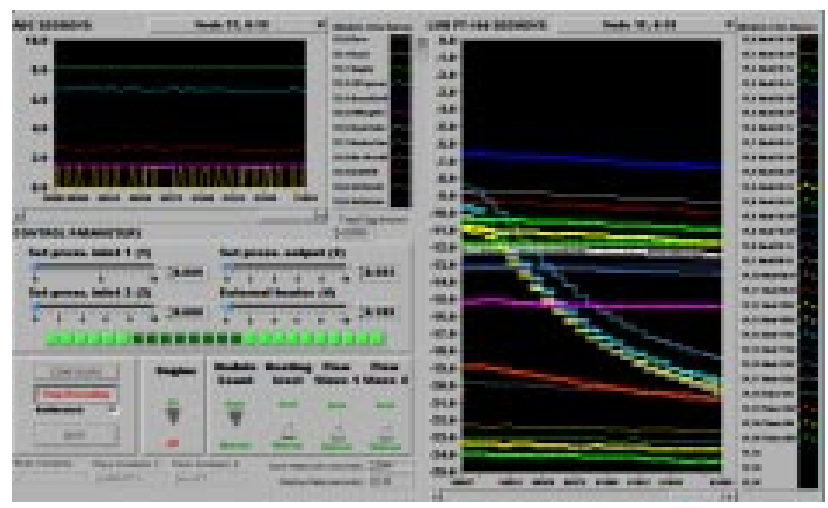

Figure 4: Typical Screen from the BridgeVIEW User Interface

Flow and pressure are controlled by commercial DACs, which are located on the same CAN bus as the LMBs. A functionally-restricted CANopen protocol allowing PDO and SDO transfers, but lacking a local object dictionary and node-guarding support, has been implemented in the form of a BridgeVIEW VI server. The server can handle both standard DS 301 PDOs, as well as the multiplexed PDOs used by the LMB. Based on this protocol, a BridgeVIEW application has been designed that permits a simplified configuration by allowing the CAN network to be scanned ${ }^{9}$ for attached CANopen devices.

Figure 4 shows a typical on line display of temperatures on the 22 SCT module thermo-structure used as a load in these tests, together with system flows and pressures

\footnotetext{
${ }^{9}$ Via Model 184726C-02 PCI-CAN/2 Communication card Mfr: National Instruments Corp, Austin TX 78759, USA
}

and the module interlock status bit pattern (square indicators).

\section{B. Hard-wired Thermal Interlocks}

\section{1) General}

In the final installation, a hard-wired thermal interlock system will automatically cut power to individual silicon pixel [7] and micro-strip modules if their temperatures exceed safe values for any reason. Cases include latch-up, de-lamination of a particular module from its cooling channel or failure of coolant flow to a particular parallel cooling circuit.

Two prototype 16 channel interlock modules ("I-Boxes") were used in combination with NTC sensors attached to the dummy silicon modules. The signals from the sensors were split between an LMB and the I-Boxes, in which comparison was made with hard-wired voltages representing the upper ("POWER DISABLE") and lower ("POWER RE-ENABLE") limits of the acceptable module temperature band.

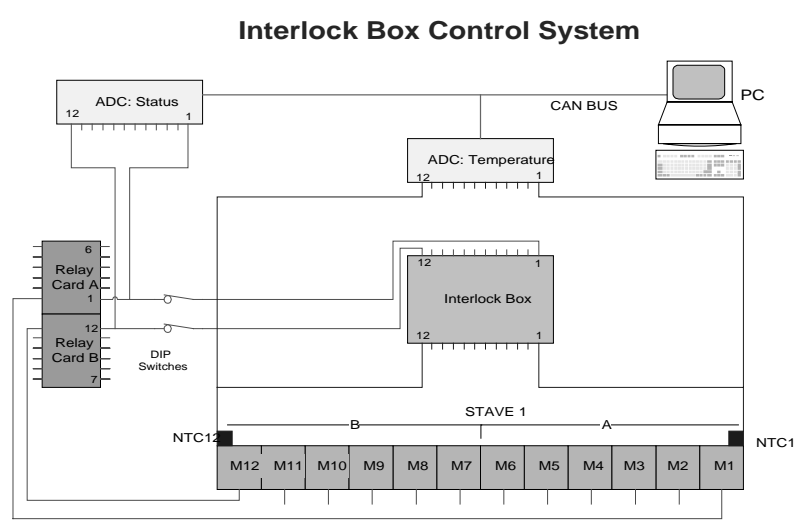

Figure 5: Interlock Study of a "Stave" of 12 Silicon modules

A second LMB was used to read the status of module power. In some of these studies (III.B.1), the counted number of module "POWER ON" status bits was used to proportionally vary the flow rate of coolant to the circuit

\section{2) The Interlock System Temperature Sensor Choice.}

As in the final experiment, the temperature sensors, which act as inputs to the I-Boxes, are mounted on the detector substrates. High radiation tolerance is required of these devices. The constraint of two-wire readout over cable lengths of up to $40 \mathrm{~m}$ predicates relatively high resistance and large $\Delta R / R$ per Kelvin. NTC resistors fulfil these requirements, and are available as $10 \mathrm{k} \Omega$ devices with $1 \%$ tolerance at $25{ }^{\circ} \mathrm{C}$ with $4 \% \Delta \mathrm{RK}^{-1}$. In the very compact region of the ATLAS pixel detector only SMD devices can be installed. Samples of NTC SMD resistors from Taiyo Yuden and Semitec were irradiated with 25 $\mathrm{GeV} / \mathrm{c}$ protons up to $1.2 \times 10^{15} \mathrm{p} / \mathrm{cm}^{2}$. Neither showed significant sensitivity to irradiation. Dispersion before and after irradiation corresponds to $\Delta \mathrm{T}< \pm 0.3 \mathrm{~K}$; within 
the acceptable maximum error limit for the sensors of $\pm 0.5 \mathrm{~K}$.

\section{RESUlTS FROM THE PRESENT STUDY}

\section{A. Studies of Heater Interlock Hysteresis}

The I-Box comparator channels are equipped with hysteresis. A fixed resistor network defines the hardwired switching temperatures. In this study, the interlock signal ("POWER DISABLE") is nominally set at $0.2^{\circ} \mathrm{C}$ and reset ("POWER RE-ENABLE") at $-0.8^{\circ} \mathrm{C}$.

To simulate the behaviour of the final power supplies in the present tests, relays were put between the power supplies and the dummy modules (Figure 5). The I-Box channels controlled these relays. Starting from stable running conditions of the cooling system a coolant runout condition was provoked. The interlock transition temperatures of all channels were recorded, and found to be centred on the nominal values within an error band of $\pm 1.0 \mathrm{~K}$, including effects of the I-Box electronics and NTC resistance. The sensors used were chosen at random, as is foreseen for the final experiment.

\section{B. Studies of Fluid Flow Proportionality}

The mass flow of coolant each circuit will be individually tuned via feedback according to the circuit load variation, using pressure regulators in the liquid supply lines. We have studied the performance of the circulator and the temperature distribution on powered silicon modules under steady state, partial-load, interlock-trip, start-up and shutdown conditions using two methods of proportional fluid control.

\section{1) I-Box Bit Counting.}

In the first study, the number of powered modules was counted via I-Box bits asserted, and the flow varied according to a protocol;

$$
\left.\mathrm{P}_{\text {CAPILLARY }}=\mathrm{P}_{\mathrm{SL}}+\mathrm{m}^{*} \text { (\#powered modules }\right)
$$

The driving pressure, $\mathbf{P}_{\text {CAPILlaRY }}$ is set by the WAGO DAC output acting through the E2P driver to the dome loaded supply regulator. The constant of proportionality $\mathbf{m}$ is the incremental pressure required to drive sufficient mass flow of $\mathrm{C}_{3} \mathrm{~F}_{8}$ liquid through the capillary to evacuate the power of a single pixel or SCT module $(\sim 10$ Watts max).

Temperature sensors and analysing heaters were mounted on the exhaust tubing downstream of the Silicon detectors to determine the amount of un-evaporated $\mathrm{C}_{3} \mathrm{~F}_{8}$. Results were good over a wide range of circuit power (number of powered modules), and indicated that very little unevaporated liquid was entering the exhaust. However, the protocol is vulnerable to variations in individual module power, unless adapted to:
$\mathrm{P}_{\text {CAPILLARY }}=\mathrm{P}_{\mathrm{SL}}+\mathrm{m} * \Sigma_{\mathrm{i}}\left(\mathrm{POWER}_{\text {module (i) }}\right)$

where $\mathbf{m}$ ' is the pressure/power conversion constant $\left(\mathrm{mbarW}^{-1}\right)$. This protocol is however dependent on continuous access to the monitored voltages and currents on several power supply rails per silicon detector module.

\section{2) Direct PID control of Fluid Flow.}

Direct PID control of circuit flow on the basis of sensed exhaust temperature has proved an effective means of control. In a first study, a commercial PID controller ${ }^{10}$ directly piloted the E2P driver. In a second study, a PID algorithm was implemented directly in a microcontroller chip ${ }^{11}$ of the same family as that used $^{12}$ for system programming and monitor functions in the recentlydeveloped "Embedded-LMB" (E-LMB)[8], currently under test. In a third study, PID control was implemented using the BridgeVIEW PID extension toolkit, using the WAGO DAC modules to pilot the E2P drivers.

In each case, it was possible to maintain the temperature at a point $\sim 50 \mathrm{~cm}$ downstream of the evaporation zone a significant margin $\left(>10^{\circ} \mathrm{C}\right)$ above the evaporation temperature, over the full range of circuit power: i.e. from one module powered to all modules powered.

Importantly also, in all cases, temperatures on powered modules stayed within allowable limits with a variation of $\pm 0.5^{\circ} \mathrm{C}$ during the transient (ramp-up/ramp-down of varying numbers of modules on the cooling circuit). The right-hand plot of Figure 4 illustrates the variation of temperature on powered and un-powered modules following a partial shut-down of six modules, with flow reduced under PID control to accommodate only the remaining powered modules. The reduction in temperature of the un-powered modules toward the evaporation (tube) temperature is seen, while the powered modules remain roughly constant in temperature. In setting up the PID parameters, care was needed to ensure that the lower pressure limit was not less than $\mathbf{P}_{\mathbf{S L}}$, during operation or PID auto-tuning. The results indicate that with proportional flow control, stability in temperature of remaining powered modules is achievable, and that a relatively simple insulation scheme will suffice to maintain the outer surface of the exhaust tubing above the local dew-point.

\section{FutURE Plans}

A $6 \mathrm{~kW}$ "demonstrator" recirculator with $\sim 25$ parallel cooling circuits is currently under construction. Its capacity is equivalent to $\sim 1 / 8$ of the barrel SCT and pixel detectors. The local control system will be modelled on

\footnotetext{
${ }^{10}$ Model G9FTE-R*E1R-88-N: Mfr: RKC Instrument Co, 16-6 Kugahara 5-Chome Ohta-Ku, Tokyo, Japan

11 AT90S8515; Mfr: ATMEL Corp, San Jose CA 95131, USA: programmed from $\mathrm{C}$ via GNU toolkit

12 ATMEL ATmega103 128k RISC flash $\mu$ controller
} 
the prototype hardware of this work, and will use ATLAS E-LMB DAQ, and the final ATLAS SCADA software.

Table 1 shows the numbers of cooling circuits, their individual power, and the number of flow and boiling pressure regulators required. The circuit count and total power resembles that of the full ATLAS SCT tracking layer 6 . The high capacity hermetic piston compressor ${ }^{13}$, its circuit flow and pressure control system represent a prototype for the first such installation at an assembly site for the ATLAS SCT and pixel detectors. In the final ATLAS installation, a total of seven such systems will be required. Several will be deployed at assembly sites before integration at CERN.

Table 1: Power and Cooling Channel Count in the Demonstrator Recirculation System

\begin{tabular}{|c|c|c|c|c|c|}
\hline Layer & Circuits & $\begin{array}{c}\text { Supply } \\
\text { Capillaries } \\
\text { / circuit }\end{array}$ & $\begin{array}{c}\text { Power/ } \\
\text { Circuit } \\
\text { (W) }\end{array}$ & $\begin{array}{c}\text { Flow } \\
\text { Regs }\end{array}$ & $\begin{array}{c}\text { Boiling. } \\
\text { Pr. Regs }\end{array}$ \\
\hline $\begin{array}{c}\text { SCT } \\
6\end{array}$ & 2 & 2 & 480 & 4 & 2 \\
\hline $\begin{array}{c}\text { SCT } \\
5\end{array}$ & 2 & 2 & 480 & 4 & 2 \\
\hline $\begin{array}{c}\text { SCT } \\
4\end{array}$ & 1 & 2 & 480 & 2 & 1 \\
\hline $\begin{array}{c}\text { SCT } \\
3\end{array}$ & 1 & 2 & 480 & 2 & 1 \\
\hline $\begin{array}{c}\text { Pixel } \\
2\end{array}$ & 4 & 1 & 208 & 4 & 4 \\
\hline $\begin{array}{c}\text { Pixel } \\
1\end{array}$ & 3 & 1 & 208 & 3 & 3 \\
\hline $\begin{array}{c}\text { B } \\
\text { layer }\end{array}$ & 2 & 1 & 144 & 2 & 2 \\
\hline $\begin{array}{c}\text { SCT } \\
\text { disk/4 }\end{array}$ & 1 & 3 & 110 & 3 & 1 \\
\hline $\begin{array}{c}\text { Pixel } \\
\text { disk/6 }\end{array}$ & 2 & 1 & 96 & 2 & 1 \\
\hline \multicolumn{2}{|c|}{ TOTALS } & 5146 & 26 & 17 \\
\hline \multicolumn{2}{|c|}{ TOTAL: SCT layer 6 } & 6720 & 28 & 14 \\
\hline
\end{tabular}

\section{REFRIGERANT IRRADIATION STUDY}

\section{1) Effects of Neutron Irradiation}

Small, static liquid samples of perfluoro-n-hexane $\left(\mathrm{C}_{6} \mathrm{~F}_{14}\right)$ were irradiated up to $3 \times 10^{13}$ fast neutrons.cm ${ }^{-2}$ to simulate the expected environment at LHC. $\mathrm{C}_{6} \mathrm{~F}_{14}$ was chosen for its chemical similarity to $\mathrm{C}_{3} \mathrm{~F}_{8}$ and for its much lower vapor pressure; a requirement in the test facilities. Studies revealed ${ }^{18} \mathrm{~F}$ to be the longest-lived radioisotope ( $\tau=106 \mathrm{~min}$ : $511 \mathrm{KeV} \gamma$ emitter). Neutron capture cross section data suggest (for an assumed instantaneous rate of $10^{6} \mathrm{n} . \mathrm{cm}^{-2} \mathrm{~s}^{-1}$ ) an activity level for ${ }^{18} \mathrm{~F}$ in the range $10^{4}-10^{5} \mathrm{~Bq} \cdot \mathrm{g}^{-1}$ during $\mathrm{C}_{3} \mathrm{~F}_{8}$ circulation. This is believed to be acceptable in a closed circuit system.

\footnotetext{
${ }^{13}$ Haug QTOGV125LM; 80m $\mathrm{hr}^{-1} \mathrm{C}_{3} \mathrm{~F}_{8}$ vapor; $\mathrm{P}_{\text {in(out) }} 1$ (10) bar ${ }_{\text {abs }}$
}

\section{2) Radiation-Induced Chemical Modifications}

Small, static liquid samples of $\mathrm{C}_{6} \mathrm{~F}_{14}$ were exposed to ${ }^{60} \mathrm{C} \gamma$ irradiation. After an absorbed dose of $3 \mathrm{Mrad}$, about $1 \%$ by weight of $\mathrm{C}_{6} \mathrm{~F}_{14}$ liquid had been radiochemically modified: there was chemical evidence of the production of reactive $\mathrm{HF}$, due to impurities containing $\mathrm{O}-\mathrm{H}$ and $\mathrm{C}-\mathrm{H}$ groups.

Water contamination can be eliminated by $3 \AA$ molecular sieves added to the cooling circuit. However, since saturated fluorocarbons $\left(\mathrm{C}_{\mathrm{n}} \mathrm{F}_{(2 \mathrm{n}+2)}\right)$, are synthesized from alcane precursors, batch testing for residual $\mathrm{H}$ contamination (using the characteristic Fourier Transform Infra-Red signature of $\mathrm{C}-\mathrm{H}$ bonds) is also advisable. Techniques for the catalytic removal of $\mathrm{C}_{n} \mathrm{~F}_{x} \mathrm{H}_{(2 n-x)}$ contamination were developed [9] for the DELPHI RICH detector, where high fluid purity is needed for good UV transparency: similar techniques can be used in the present application.

Scanning electron microscopy and Auger electron spectroscopy identified $\mathrm{C}, \mathrm{F}$ and $\mathrm{O}$ - containing polymeric film deposits, formed on stainless steel and aluminum samples immersed in liquid $\mathrm{C}_{6} \mathrm{~F}_{14}$ during irradiation. After 6Mrad, surfaces were almost uniformly covered to a depth of $\sim 0.4 \mu \mathrm{m}$. Degradation and plate-out were greater in a sample of $\mathrm{C}_{6} \mathrm{~F}_{14}$ to which $3 \%$ (vol.) n-heptane had been added to act as a $\mathrm{H}$-source.

\section{CONCLUSIONS}

We have developed the control system for a multiple parallel channel $\mathrm{C}_{3} \mathrm{~F}_{8}$ evaporative cooling recirculator. Power interlocks and proportional control of refrigerant flow have been successfully demonstrated. During transient conditions, powered modules remained stable in temperature, while the exhaust tubing of the evaporative cooling circuits could be stably maintained a significant margin above the $\mathrm{C}_{3} \mathrm{~F}_{8}$ evaporation temperature, (at a higher temperature than possible in a liquid cooling system), simplifying the insulation in the final ATLAS installation.

The use of $\mu$ controller-based PID algorithms for flow control is particularly interesting, and offers the possibility of implementation of either smart (PID) DACs or "transparent" DACs in the new ATLAS DCS embedded local monitor board.

Irradiation studies have indicated that generic saturated fluoro-carbon refrigerants of the form $\left(\mathrm{C}_{n} \mathrm{~F}_{(2 n+2)}\right)$ are suited to the ATLAS application provided hydrogen donor impurities are kept at a low level using standard purification procedures. 


\section{REFERENCES}

[1] E. Anderssen et al, "Fluorocarbon Evaporative Cooling Developments for the ATLAS Pixel and Semiconductor Tracking Detectors",

Proc. $5^{\text {th }}$ workshop on electronics for LHC experiments, CERN 99-09 CERN/LHCC/99-33, pp 421-426.

[2] V. Vacek et al, "Per-fluorocarbons and their use in Cooling Systems for Semiconductor Particle Detectors" Accepted for publication in Fluid Phase Equilibria

[3] "Controller area network (CAN) for high-speed communication” ISO 11898 (1993)

[4] B. Hallgren et al, "A Low Cost I/O Concentrator Using the CAN Field-Bus", CERN-EP-99-159, Oct 1999 Proc. International Conference on Accelerator and Large Experimental Physics Control Systems, Trieste, Italy, 4-8 Oct 1999
[5] DS 301, CANopen Application Layer and Communication Profile Version 4.0, June 2000, CiA, CAN in Automation e. V.

[6] CANopen Working Draft 404 "Device Profile for Measuring Devices and Closed Loop Controllers".

[7] See for example: ATLAS Pixel Detector Technical Design Report CERN/LHCC/98-13, 31.05.1998 (Chapter 9)

[8] ATLAS Embedded Local Monitor Board ("E-LMB") http://atlasinfo.cern.ch/ATLAS/GROUPS/DAQTRIG/ DCS/LMB/SB/elmb_1.html

[9] S. Ilie \& G. Lenzen, "Per-fluorocarbon Liquid: Specific Chemical Aspects for use within the DELPHI RICH" (DELPHI internal report) DELPHI 93-33 RICH54, 30 March 1993. 